\section{An exclusive new range of innovative brushes}

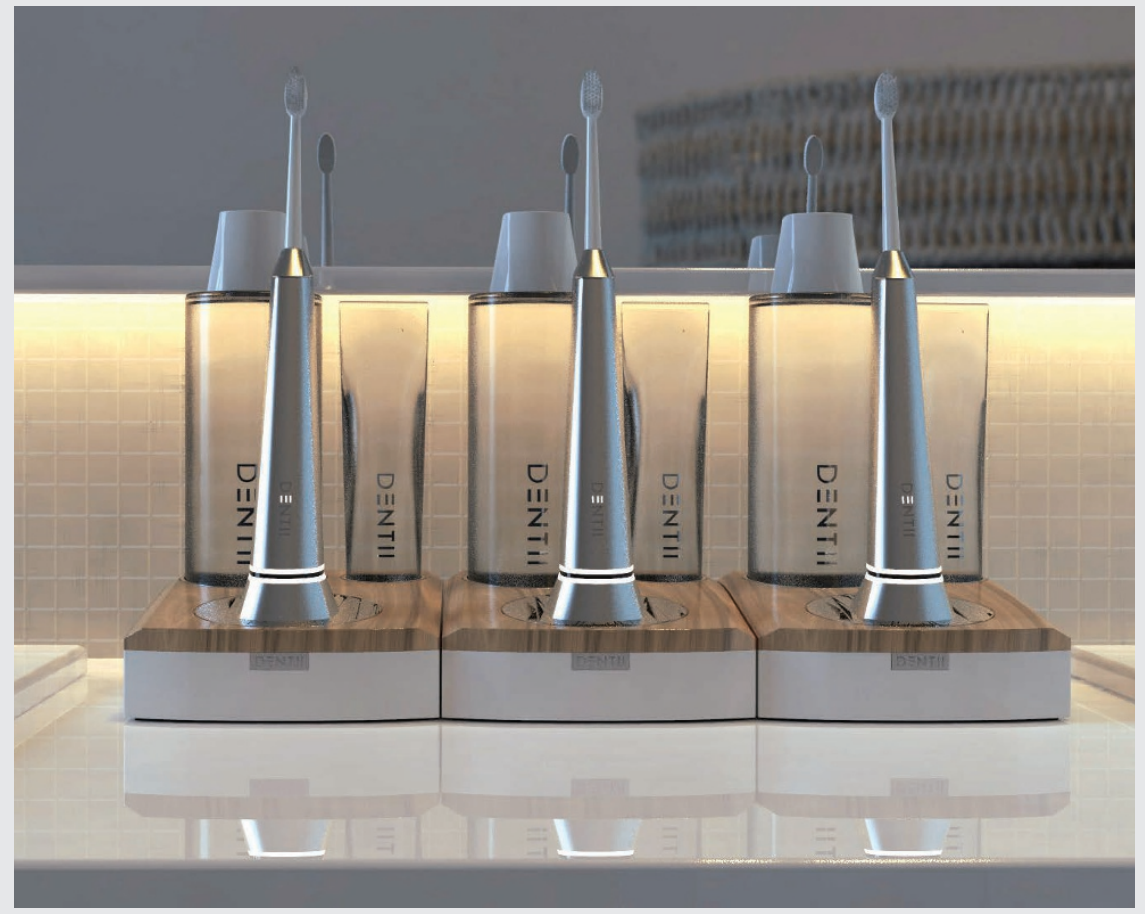

London dentist and entrepreneur Dr Sunny Sihra has created a new, integrated oral care system called Dentii.

Founded by Dr Sihra and developed by business partner David Fowler from City of London-based INDEV Innovation Developments, Dentii comprises top quality, sleek electronic toothbrushes with single-use biodegradable brush heads at the core of the exclusive range as well as being the world's most innovative brush.

The electric toothbrushes are manufactured in anodised aluminium and precious metal finishes including Silver, Gold and Rose Gold editions for maximum sophistication. A bespoke range of wooden finishes complete the look.

As back-up for those who travel a great deal, manual toothbrushes and travel cases are available in a range of wood finishes.

When connected to a smartphone app, the electric toothbrush maps the geometry of a patient's mouth, noting the brushing paths and pressure. These data can then be accessed by the user and, if they wish, their dentist, leading to better overall dental hygiene. The toothbrush is being developed as the world's first brush that can prompt you to go to the dentist at early signs of problems, and with patented technology Dr Sihra is keen to develop this into the world's first oral cancer screening tool.

Dentii uses a single-use, biodegradable brush head which is interchangeable each time you brush. The disposable brush head is revolutionising the hygiene issues found in multiple-use brush heads, namely the daily increased contamination by bacteria, fungus and chemicals typically found in bathrooms. The whole system is subscription-based so you never run out of a fresh supply of brush heads, toothpaste and mouthwash.

The brush heads are made of natural materials that break down over time and can be disposed of in the same way as discarded food, or simply composted.

The brand is offering multiple subscription levels from base level packages providing single head support per day through to prescription level with two brush heads per day. Prescription toothpastes, mouthwashes, interdental brushes and carry cases complete the range.

Pricing starts at $£ 99.99$ and increases to $£ 299.99$ depending on the level of the subscription package and type of brush.

The new Dentii range is being launched on Kickstarter in February 2018, with the first toothbrushes available at the end of the year. http://dentii.co.uk/

\section{A new premium prophylaxis system}

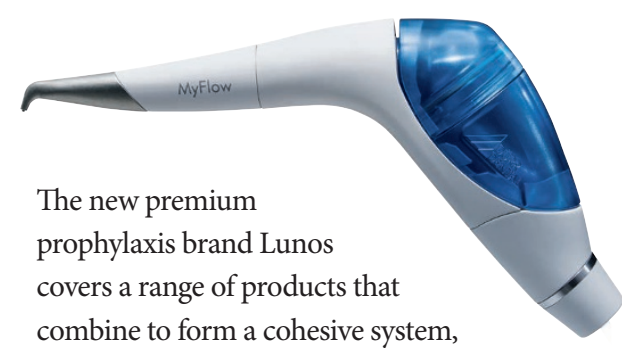

offering unique advantages over existing products.

One example of this is the MyFlow powder jet handpiece. Its unique exchangeable chamber principle means the powder container can be replaced quickly and easily, avoiding the inconvenience of having to refill in the middle of treatment. Furthermore, surgeries can prepare enough powder containers for the whole day.

The powder jet handpiece also sets new standards in terms of reprocessing. All parts are thermally disinfectable and autoclavable. Together with the minimised clogging potential and ease of maintenance, this saves time, taking pressure off your treatment workload.

MyFlow works with various prophylaxis powders. The Gentle Clean variant of Lunos contains innovative new abrasive agents based on the non-carcinogenic disaccharide trehalose for gentle cleaning in the supragingival area and is available in three different flavours. Alternatively, there's Lunos prophy powder Perio Combi for supragingival and subgingival treatments. The excellent water solubility of this powder enables safe, virtually residue-free dissolution in the periodontal pocket and suction system. Thanks to this, patients no longer experience the unpleasant grittiness typically associated with this type of product.

There are also two variants of polishing paste available. The abrasive particles in the Lunos prophy paste Two in One become smaller during the polishing process ensuring that coatings and plaque are removed without interfering with the surfaces of restorations. Even gentler polishing is possible with the Lunos prophy paste Super Soft. Its low RDA value makes it suitable for sensitive surfaces, for example on children or patients with implants. The prophy pastes from Lunos are also available in different flavours.

Also, included in the Lunos range is an alcohol-free rinse solution, a fluoride varnish, fluoride gel, and fissure sealant. Lunos is a new sub-brand of Dürr Dental. For more information visit www.duerrdental.com. 